\title{
Search for single production of the vector-like top partner at the 14 TeV LHC
}

\author{
Yao-Bei Liu ${ }^{\text {a }}$, Yu-Qi Li \\ Henan Institute of Science and Technology, Xinxiang 453003, People's Republic of China
}

Received: 24 May 2017 / Accepted: 15 September 2017 / Published online: 30 September 2017

(C) The Author(s) 2017. This article is an open access publication

\begin{abstract}
The new heavy vector-like top partner $(T)$ is one of typical features of many new physics models beyond the standard model. In this paper we study the discovery potential of the LHC for the vector-like $T$-quark both in the leptonic $T \rightarrow b W$ and $T \rightarrow t_{\text {lep }} Z_{\text {lep }}$ (trilepton) channels at $\sqrt{s}=14$ $\mathrm{TeV}$ in the single production mode. Our analysis is based on a simplified model including a $S U(2)_{L}$ singlet with charge $2 / 3$ with only two free parameters, namely the $T W b$ coupling parameter $g^{*}$ and the top-partner mass $m_{T}$. The $2 \sigma$ exclusion limits, $3 \sigma$ evidence and the $5 \sigma$ discovery reach in the parameter plane of $g^{*}-m_{T}$, are, respectively, obtained for some typical integrated luminosity at the $14 \mathrm{TeV}$ LHC. Finally we analyze the projected sensitivity in terms of the production cross section times branching fraction for two decay channel.
\end{abstract}

\section{Introduction}

Various extensions of the standard model (SM) predict new heavy particles that address the hierarchy problem caused by the quadratic divergences in the quantum-loop corrections to the Higgs boson mass; for a review see [1]. The largest corrections, owing to the top-quark loop, are canceled by the existence of heavy partners of the top quark in many of these models, such as little Higgs [2,3], extra dimensions [4] and composite Higgs [5,6] models. The discovery of the $125 \mathrm{GeV}$ Higgs boson $[7,8]$ at the Large Hadron Collider (LHC) has excluded heavy SM-like chiral fermions [9]. Therefore, we focus on the case of a $S U(2)_{L}$ singlet vector-like top partner $(T)$. The effects on Higgs production and decay rates of loop diagrams including $T$ quarks are well below the precision of the current measurements [10-16]. In many cases, the vector-like top partners mix with the SM quarks predominantly of the third generation and can also stabilize the electroweak vacuum $[17,18]$. The phenomenology of new heavy quarks has been widely studied in the literature; see

a e-mail: liuyaobei@hist.edu.cn for example [13,19-81] and the forthcoming direct searches at the LHC will therefore play an important role in testing many models predicting the existence of these states.

The current combined results of ATLAS and CMS searches have established lower limits on mass of the vectorlike top partners in the range of $550-900 \mathrm{GeV}$ at center-ofmass energies of $8 \mathrm{TeV}$ [82-86] and $13 \mathrm{TeV}$ [87-89], depending on the assumed branching ratios. Most of the experimental searches assume the top partners to be pair produced via the strong interaction, and these bounds strongly depend on the assumptions on the decay branching ratios and the properties of the top partner. Because the vector-like quarks can induce corrections to precisely measured observables of the SM, the relevant model parameters can also be constrained by the indirect searches of the electroweak precision observables [90-96]. On the other hand, it is possible that the new vector-like top partners can significantly mix with the SM light quarks [97-105]. However, such indirect constraints on the mixing parameters may be relaxed if several multiplets are present in the low-energy spectrum [106]. For high $m_{T}$ (about $m_{T} \gtrsim 1 \mathrm{TeV}$ ), previous study showed that single production of top partners starts to dominate over pair production due to larger phase space $[66,67,107-114]$. Especially for the case that the top partners can mix in a sizable way with lighter quarks, their production cross section will be very large due to the mixing with valence quarks [115-119]. We do not consider this case because the masses of the top partners are not connected to electroweak symmetry breaking.

Very recently, both the ATLAS [120] and the CMS [121] Collaborations presented a search optimized fora single produced vector-like $T$ quark at $\sqrt{s}=13 \mathrm{TeV}$, subsequently decaying as $T \rightarrow W b$ with leptonic decays of the $W$ boson. The smallest coupling limit on the $T W b$ coupling strength has been set as $\left|c_{L}^{W b}\right|=0.45$ for a vector-like top partner with a mass of $1 \mathrm{TeV}$ [120]. This encouraged us to further analyze this process in order to provide an effective search strategy for the future $14 \mathrm{TeV}$ LHC. In particular, we also studied the 
observational potential of single vector-like top partner in the $T \rightarrow t_{\text {lep }} Z_{\text {lep }}$ decay channel at $14 \mathrm{TeV}$ high-luminosity (HL)-LHC with an integrated luminosity of $3 \mathrm{ab}^{-1}$. Although the branching ratio of the $T \rightarrow t_{\text {lep }} Z_{\text {lep }}$ (about $0.37 \%$ ) decay channel is small and results in a suppressed production rate for the final states, it has the great advantage of small QCD backgrounds [122,123]. Therefore, we here mainly study the observability of a single $T$-quark production at the $14 \mathrm{TeV}$ LHC both for the leptonic $T \rightarrow b W$ and for the $T \rightarrow t_{\text {lep }} Z_{\text {lep }}$ (trilepton) channels, and we discuss the event selection and cuts on kinematic variables in detail. Finally, the exclusion limits and discovery potential of the production cross section times branching fraction for two decay channels are, respectively, examined as a function of top-partner mass for the several typical luminosity at the LHC. In order to keep the model as independent as possible, we here perform the study in the framework of a simplified model, which only comprises two independent parameters.

The rest of the paper is organized as follows. In Sect. 2 we briefly describe the main features of the simplified model. In Sect. 3 we turn to the study of the prospects of observing the single $T$ production by performing a detailed analysis of the signal and backgrounds in both the leptonic $W b$ and the $T \rightarrow t_{\text {lep }} Z_{\text {lep }}$ decay channels at $14 \mathrm{TeV}$ LHC. Finally, we conclude in Sect. 4.

\section{Top partner in the simplified model}

As mentioned above, the benefit of using of simplified models is that the results of the studies could be used to make predictions for more complex models including the top partners. A generic parametrization of an effective Lagrangian for top partners has been proposed in Ref. [105], where the vector-like quarks are embedded in different representations of the weak $S U$ (2) group. We here consider a simplified model where the vector-like $T$-quark is an $S U(2)$ singlet with charge $2 / 3$, with couplings only to the third generation of SM quarks. The benefit of using the simplified effective theory is that the results of the studies could be used to make predictions for more complex models including various types of top partners.

The top-partner sector of the model is described by the general effective Lagrangian (showing only the couplings relevant for our analysis) [105]

$$
\begin{aligned}
\mathcal{L}_{T}= & \frac{g^{*}}{\sqrt{2}}\left[\frac{g}{\sqrt{2}} \bar{T}_{L} W_{\mu}^{+} \gamma^{\mu} b_{L}+\frac{g}{2 \cos \theta_{W}} \bar{T}_{L} Z_{\mu}^{+} \gamma^{\mu} t_{L}\right. \\
& \left.-\frac{m_{T}}{v} \bar{T}_{R} h t_{L}-\frac{m_{t}}{v} \bar{T}_{L} h t_{R}\right]+ \text { h.c. },
\end{aligned}
$$

where $m_{T}$ is the top-partner mass, and $g^{*}$ parametrizes the single production coupling in association with a $b$ - or a top-

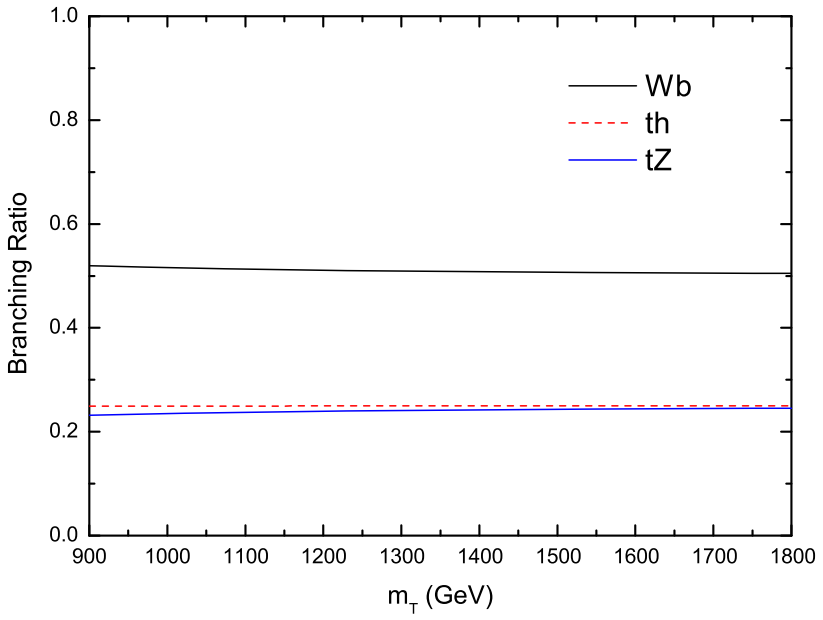

Fig. 1 Branching ratios for the three decay modes for various of toppartner masses with $g^{*}=0.2$

quark. $g$ is the $S U(2)_{L}$ gauge coupling constant, $v \simeq 246$ $\mathrm{GeV}$ and $\theta_{W}$ is the Weinberg angle. Thus there are only two model parameters: the top-partner mass $m_{T}$ and the coupling strength to SM quarks in units of standard couplings, $g^{*}$. Here we take a conservative range for the coupling parameter [120,124]: $g^{*} \leq 0.5$, which is consistent with the current experiment bounds.

In general, the vector-like $T$-quark has three different decay channels into SM particles: $b W, t Z$, and $t h$. In Fig. 1, we show the branching ratios of three decay channels by varying top-partner masses with $g^{*}=0.2$. We can see that $\operatorname{Br}(T \rightarrow t h) \approx \operatorname{Br}(T \rightarrow t Z) \approx \frac{1}{2} \operatorname{Br}(T \rightarrow W b)$ is a good approximation as expected by the Goldstone boson equivalence theorem [125].

\section{Event generation and analysis}

In this section, we analyze the observation potential by performing a Monte Carlo simulation of the signal and background events and explore the sensitivity of single top partner at the LHC through $T \rightarrow b W$ and $T \rightarrow t Z$ channels. The Feynman diagram of the production and decay chain is presented in Fig. 2.

The model file generating signal events according to the Lagrangian of Eq. (1) can be found in the dedicated FeynRules [126] model database webpage [127]. The SM input parameters relevant in our study are taken from [128]. The corresponding free parameters are the top-partner mass $m_{T}$ and the coupling parameter $g^{*}$, which governs the top-partner single production involving a $t$-channel $W$ boson. Considering the current constraints from the top-partner pair production processes at $13 \mathrm{TeV}$ ATLAS detector [87-89], we generate eight benchmark points varying the $T$ mass in steps of $100 \mathrm{GeV}$ in the range $m_{T} \in[900 ; 1800] \mathrm{GeV}$ with $g^{*}=0.2$. 


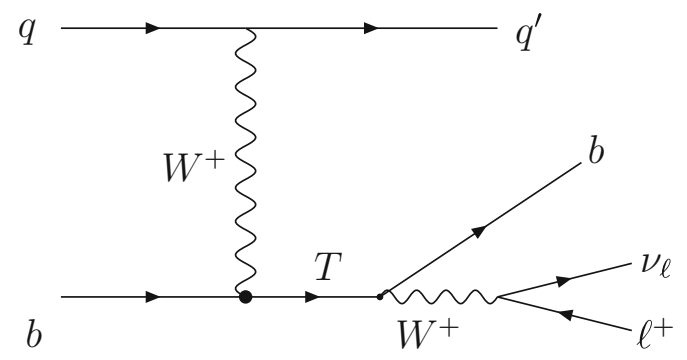

(a)

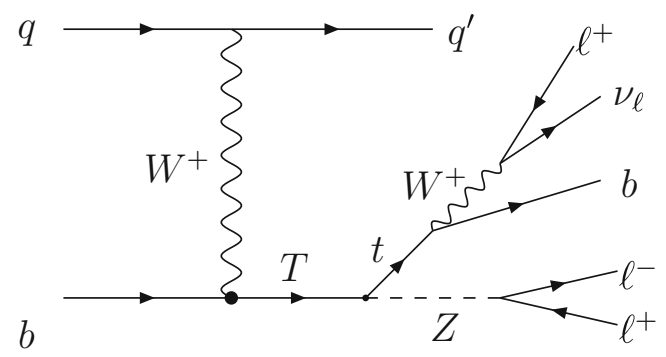

(b)

Fig. 2 The Feynman diagram for production of single $T$ quark including the decay chains $T \rightarrow b W\left(\rightarrow \ell^{+} \nu\right)$ and $T \rightarrow t\left(\rightarrow b \ell^{+} \nu\right) Z\left(\rightarrow \ell^{+} \ell^{-}\right)$

The QCD next-to-leading order (NLO) prediction for the single top-partner productions are calculated in Refs. [113,129]. Following Ref. [129], we here take the conservative value of the $K$-factor as 1.2 for the signal before the event generation.

Signal and background events are simulated at the leading order using MadGraph5-aMC@NLO [130] with the CTEQ6L parton distribution function (PDF) [131] and the renormalization and factorization scales are set dynamically by default. Pythia6 [132] and Delphes [133] are used to perform the parton shower and the fast detector simulations, respectively. The anti- $k_{t}$ algorithm [134] with parameter $\Delta R=0.4$ is used to reconstruct the jets. Event analysis is performed by using the program of MadAnalysis5 [135].

\subsection{The $T \rightarrow W b$ channel}

In this section, we analyze the observation potential by performing a Monte Carlo simulation of the signal and background events and explore the sensitivity of single top partner at the LHC through the channel

$p p \rightarrow T\left(\rightarrow b W^{+}\right) j \rightarrow b W^{+}\left(\rightarrow \ell^{+} \bar{v}_{\ell}\right) j$

For this channel, the typical signal is exactly one charged lepton, one $b$ jet, one forward jet and missing energy. The dominant background turns out to be the $W+$ light jets with one of the jets misidentified as $b$-quark jet and $t \bar{t}$ (semileptonic) + jets. $W+b+$ light jets and $W+b \bar{b}$ can also make contributions to the backgrounds. Meanwhile, the $t \bar{t}$ samples are normalized to the theoretical cross-section value for the inclusive $t \bar{t}$ process of $953.6 \mathrm{pb}$ performed at next-tonext-to-leading order (NNLO) in QCD and including resummation of next-to-next-to-leading logarithmic (NNLL) soft gluon terms [136]. The QCD corrections for the dominant backgrounds are considered by including a $k$ factor, which is 1.12 for $W^{+} b j$ [137], 1.5 for $W^{+} b \bar{b}$ [137], and about 1.2 for $W^{+} j j$ [138-142]. On the other hand, the MLM matching scheme is used, where we included up to three extra jets for $W_{\text {lep }}+$ jets and up to one additional jet to $t \bar{t}$ in the simulations [143]. Other smaller backgrounds come from single top $(t W, t$-channel and $s$-channel with up to one additional jet)

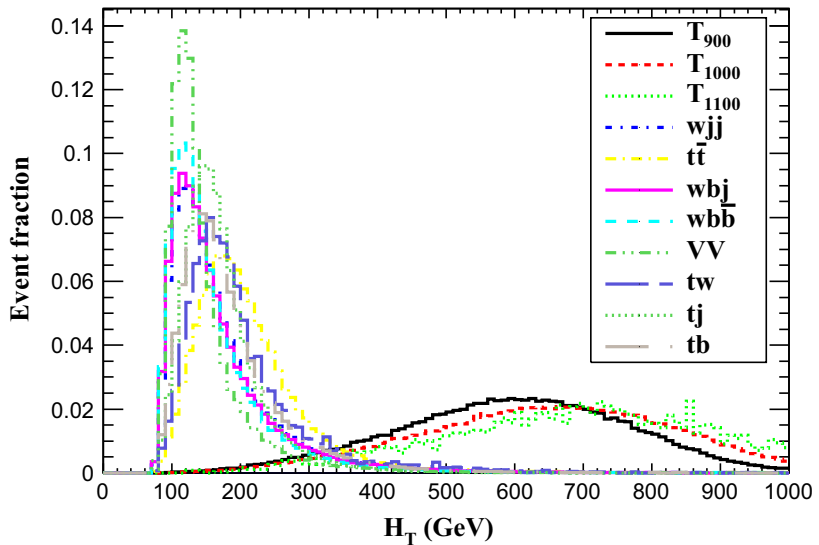

Fig. 3 Normalized scalar sum of the transverse momenta $H_{T}$ for the signals and backgrounds

and diboson ( $W W, Z Z, W Z$ ) production. The cross sections are scaled according to the approximate NNLO theoretical predictions [144, 145, 145, 146].

In our simulation, all signal and background events are required to pass the following basic cuts:

- There is exactly one isolated electron or muon $\left(N_{\ell}=1\right)$ with $p_{T}^{\ell}>25 \mathrm{GeV}$ and $\left|\eta_{\ell}\right|<2.5$.

- Jets are required to satisfy $p_{T}^{b}>25 \mathrm{GeV}$ and $\left|\eta_{b}\right|<5.0$. There is exactly one b-tagged jet $\left(N_{b}=1\right)$ with $p_{T}^{b}>$ $25 \mathrm{GeV}$ and $\left|\eta_{b}\right|<2.5$ and there are no more than three jets in total $\left(N_{j}<3\right)$.

- The missing transverse momentum $\mathbb{E}_{T}^{\text {miss }}$ is required to be larger than $20 \mathrm{GeV}$.

In order to choose appropriate kinematic cuts, we show some important kinematic distributions for the signal and the backgrounds. In Fig. 3, we show the normalized distribution of the signals and backgrounds on $H_{T}$, defined as the scalar sum of the transverse momenta of the $b$-tagged jet, the untagged jet and the lepton. From the figure, we can see that the distributions of $W j j, t \bar{t}$, single top and diboson backgrounds have peaks below $200 \mathrm{GeV}$, while the peak positions 

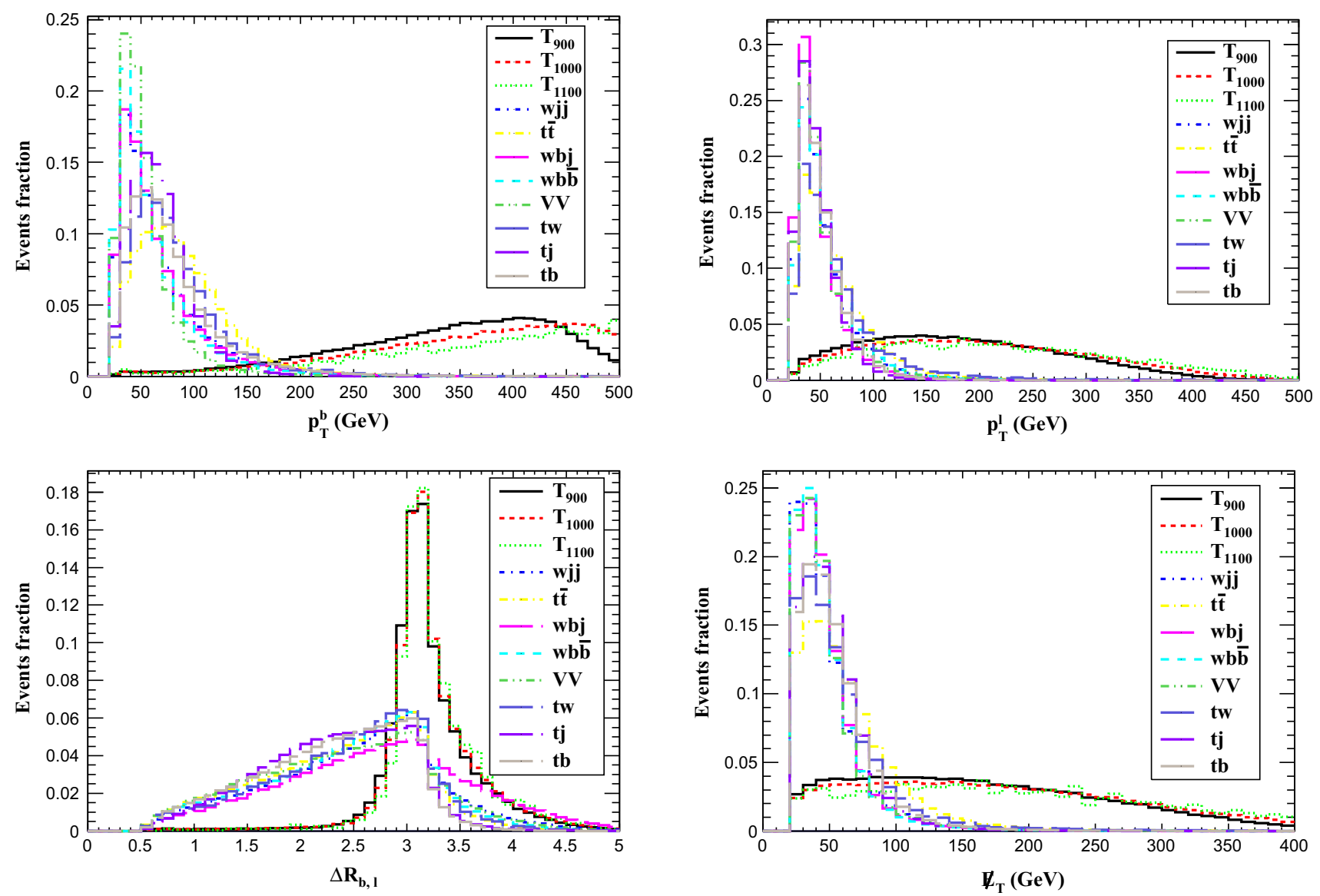

Fig. 4 Normalized distributions of the transverse momenta $\left(p_{T}^{b}\right.$ and $\left.p_{T}^{\ell}\right), \Delta R_{b, \ell}$ and $\mathbb{E}_{T}$ for the signals and backgrounds

of the signals are larger than $500 \mathrm{GeV}$. Thus we choose the $H_{T}$ cut as follows.

- Cut 1: $H_{T}>500 \mathrm{GeV}$.

In Fig. 4, we show the normalized distributions of the transverse momenta $p_{T}^{\ell}, p_{T}^{b}$, the variable $\Delta R(b, \ell)$ and the missing transverse momentum $\mathbb{E}_{T}$ for the signals and backgrounds. Here $\Delta R=\sqrt{(\Delta \phi)^{2}+(\Delta \eta)^{2}}$ is the particle separation among the objects (the tagged $b$-jet and the lepton) in the final state with $\Delta \phi$ and $\Delta \eta$ being the separation in the azimuth angle and rapidity, respectively. In the decay of a singly produced top partner, the lepton from the leptonic $W$ boson decay and the $b$ quark tend to be produced with the transverse momenta pointing in opposite directions. On the other hand, since the $W$ boson originating from heavy top-partner decay has significant transverse momentum $p_{T}$, events are required to have large missing energy due to the undetected neutrino from the $W$ boson decay. Based on these kinematical distributions, we impose the following cuts to get a high significance.

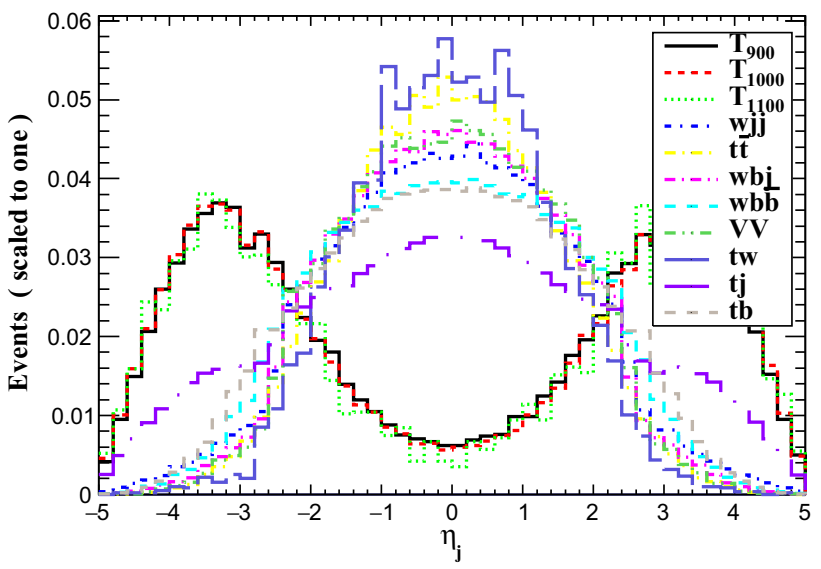

Fig. 5 Normalized distribution of the rapidity of the forward jet for the signals and backgrounds

- Cut 2: $p_{T}^{\ell}>100 \mathrm{GeV}, p_{T}^{b}>250 \mathrm{GeV}, 2.8<$ $\Delta R(b, \ell)<3.5$ and $\mathbb{E}_{T}>100 \mathrm{GeV}$.

Since the jet from splitting of a valence quark with one $W$ emission always has a strong forward nature, we plot the distribution of the rapidity of the forward jet in Fig. 5 for 


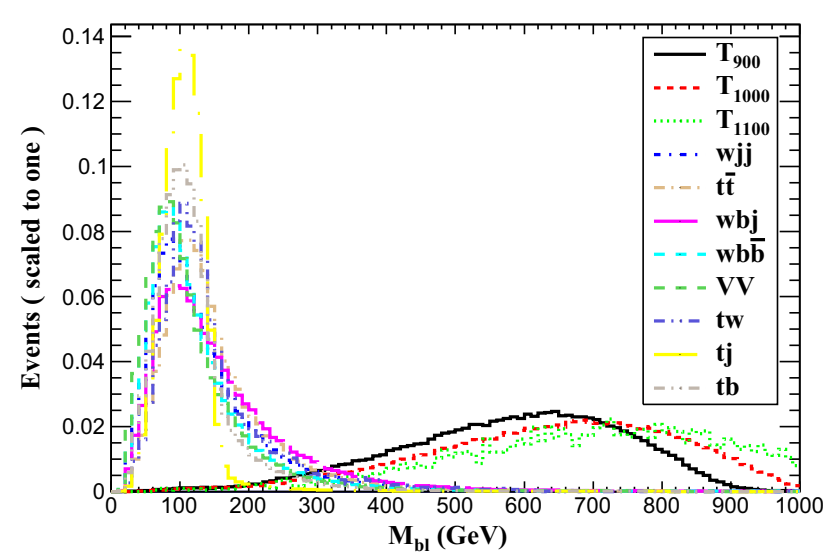

Fig. 6 Normalized invariant mass distribution of $b \ell$ system for the signals and backgrounds

the signals and backgrounds. From these distributions we can efficiently reduce the backgrounds by requiring the light untagged jet to have the following cut.

- Cut $3:\left|\eta_{j}\right|>2.4$.

The invariant mass of the $b$-tagged jet and the lepton is plotted in Fig. 6 for the signals and the backgrounds. One can see that the signal distributions peak close to the $T$-quark mass, while background distributions turn over at lower masses. Thus we can further reduce the backgrounds by the following cut.

\section{- Cut 4: $M_{b \ell}>500 \mathrm{GeV}$.}

We present the cross sections of three typical signal $\left(m_{T}=900,1000,1100 \mathrm{GeV}\right)$ and the relevant backgrounds after imposing the cuts in Table 1. From Table 1, one can see that all the backgrounds are suppressed very efficiently after imposing the selections. From the numerical results as listed in the last line of Table 1 , one can see that $W^{+} j j$ is the most dominant background after applying all those mentioned cuts. To estimate the observability quantitatively, we adopt the significance measurement [147]:

$S S=\sqrt{2 \mathfrak{i n t}_{\mathrm{int}}\left[\left(\sigma_{S}+\sigma_{B}\right) \ln \left(1+\sigma_{S} / \sigma_{B}\right)-\sigma_{S}\right]}$,

where $\sigma_{S}$ and $\sigma_{B}$ are the signal and background cross sections and $f_{\text {int }}$ is the integrated luminosity. Here we define the discovery significance as $S S=5$, the possible evidence as $S S=3$ and the exclusion limits as $S S=2$. We do not consider the theoretical and systematic uncertainties, such as the choice of the PDF set, the renormalization and factorization scales and the respective normalization to the theoretical NNLO cross sections, but we expect this will not change our results significantly.
In Fig. 7, the $2 \sigma, 3 \sigma$ and $5 \sigma$ lines are drawn as a function of $g^{*}$ and the top-partner mass $m_{T}$ for two fixed values of the integrated luminosity: 100 and $300 \mathrm{fb}^{-1}$. We can see that, for $m_{T}=0.9$ (1.6) TeV, the $5 \sigma$ level discovery sensitivities of $g^{*}$ are, respectively, about $0.14(0.34)$ with $£_{\text {int }}=100 \mathrm{fb}^{-1}$ and $0.10(0.22)$ with $£_{\text {int }}=300 \mathrm{fb}^{-1}$. On the other hand, from the $2 \sigma$ exclusion limits one can see that, for $m_{T}=1.0(1.5) \mathrm{TeV}$, the upper limits on the size of $g^{*}$ are, respectively, given as $g^{*} \leq 0.12(0.22)$ with $£_{\text {int }}=100 \mathrm{fb}^{-1}$ and $g^{*} \leq 0.09(0.16)$ with $£_{\text {int }}=300 \mathrm{fb}^{-1}$.

\subsection{The $T \rightarrow t Z$ channel}

Next, we analyze the observation potential and explore the sensitivity of the single $T$-quark at the $14 \mathrm{TeV}$ LHC through the channel

$p p \rightarrow T(\rightarrow t Z) j \rightarrow t\left(\rightarrow b \ell^{+} \bar{v}_{\ell}\right) Z\left(\rightarrow \ell^{+} \ell^{-}\right) j$.

The main SM backgrounds that can give three leptons in the final state are $t \bar{t}, W Z j j, t \bar{t} V(V=Z / W)$ and the irreducible $t Z j$. In the $t \bar{t}$ case (both top quarks decay semileptonically), a third lepton comes from a semi-leptonic Bhadron decay in the b-jet. Here we do not consider multijet backgrounds where jets can be faked as electrons, since they are very negligible in multilepton analyses [148].

First of all, we apply the following cuts on the signal and background events.

- Basic cuts: $p_{T}^{\ell, b}>25 \mathrm{GeV}, p_{T}^{j}>40 \mathrm{GeV},\left|\eta_{\ell, b}\right|<2.5$, $\left|\eta_{j}\right|<5$, where $\ell=e, \mu$.

Further, we apply some general preselections as follows.

- Cut-1: There are exactly three isolated leptons $(N(\ell) \equiv$ $3)$, at least two jets and no more than three $(2 \leq N(j) \leq$ $3)$, of which exactly one is b-tagged $(N(b) \equiv 1)$.

The requirement of three leptons can strongly reduce the $t \bar{t}$ backgrounds, and the b-tagging can efficiently suppress the diboson components.

Based on the kinematical distributions of the signal and backgrounds in Fig. 8, we furthermore impose the following cuts to get a high significance.

- Cut-2: The transverse momenta of the leading and subleading leptons from the $Z$ boson are required to have $p_{T}^{\ell_{1}}>150 \mathrm{GeV}$ and $p_{T}^{\ell_{2}}>80 \mathrm{GeV}$, and the invariant mass of the $Z$ boson is required to have $\left|M\left(\ell_{1} \ell_{2}\right)-m_{Z}\right|<$ $15 \mathrm{GeV}$.

- Cut-3: The transverse mass of the top quark is reconstructed as $140 \mathrm{GeV}<\mathrm{M}_{\mathrm{T}}\left(\mathrm{b} \ell_{3}\right)<190 \mathrm{GeV}$.

- Cut-4: We require the light untagged jet to have $\left|\eta_{j}\right|>$ 2.4 . 
Table 1 The cut flow of the cross sections (in fb) for three typical signals and the relevant backgrounds at the $14 \mathrm{TeV}$ LHC. Here we take the parameter as $g^{*}=0.2$

\begin{tabular}{|c|c|c|c|c|c|c|c|c|c|c|c|}
\hline \multirow[t]{2}{*}{ Cuts } & \multicolumn{3}{|l|}{$\underline{\text { Signal }}$} & \multirow[t]{2}{*}{$W^{+} j j$} & \multirow[t]{2}{*}{$t \bar{t}$} & \multirow[t]{2}{*}{$W^{+} b j$} & \multirow[t]{2}{*}{$W^{+} b \bar{b}$} & \multirow[t]{2}{*}{$V V$} & \multirow[t]{2}{*}{$t W$} & \multirow[t]{2}{*}{$t j$} & \multirow[t]{2}{*}{$t \bar{b}$} \\
\hline & $900 \mathrm{GeV}$ & $1000 \mathrm{GeV}$ & $1100 \mathrm{GeV}$ & & & & & & & & \\
\hline Basic cuts & 2.84 & 1.96 & 1.58 & 2012 & 106 & 238 & 361 & 27 & 97 & 3066 & 166 \\
\hline Cut 1 & 1.96 & 1.48 & 1.16 & 17.1 & 1.7 & 2.2 & 2.1 & 0.1 & 1.84 & 7.7 & 1.3 \\
\hline Cut 2 & 1.24 & 0.92 & 0.72 & 2.19 & 0.37 & 0.12 & 0.23 & 0.02 & 0.27 & 0.09 & 0.14 \\
\hline Cut 3 & 0.88 & 0.68 & 0.52 & 0.4 & 0.015 & 0.015 & 0.04 & 0.004 & 0.027 & 0.012 & 0.023 \\
\hline Cut 4 & 0.76 & 0.64 & 0.48 & 0.31 & 0.011 & 0.012 & 0.036 & 0.002 & 0.024 & 0.006 & 0.013 \\
\hline
\end{tabular}
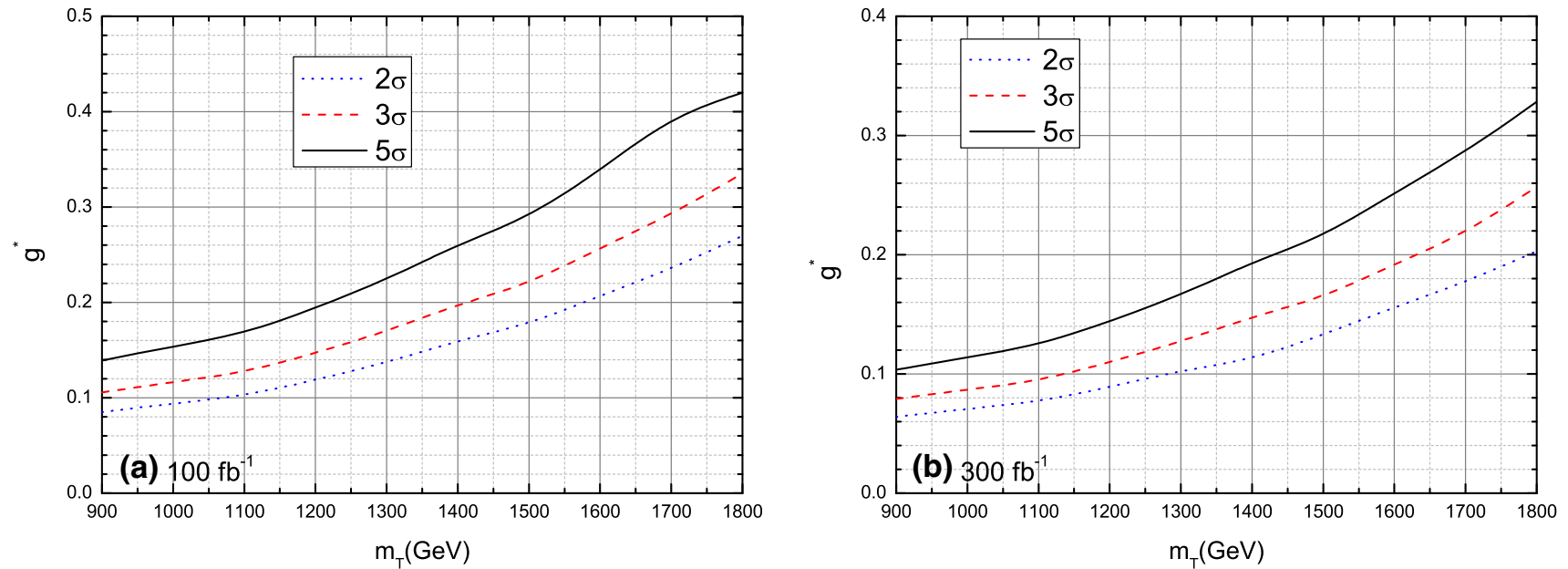

Fig. 7 The $2 \sigma, 3 \sigma$ and $5 \sigma$ contour plots for the signal in $g^{*}-m_{T}$ at $14 \mathrm{TeV}$ LHC with two typical values of integrated luminosity: a $100 \mathrm{fb}^{-1}$, b $300 \mathrm{fb}^{-1}$

- Cut-5: The transverse mass of the top-partner decay products $3 \ell b$ is required to have $M_{T}(b 3 \ell)>800 \mathrm{GeV}$.

We present the cross sections of four typical signal $\left(m_{T}=\right.$ $900,1000,1100,1200 \mathrm{GeV}$ ) and the relevant backgrounds after imposing the cuts in Table 2. From Table 2, one can see that all the backgrounds are suppressed very efficiently after imposing the selections. For the integrated luminosity $f_{\text {int }}=3 \mathrm{ab}^{-1}$, the number of events for total backgrounds after Cut-5 is found to be about six, while for the signal we obtain about 33 events for $m_{T}=1 \mathrm{TeV}$ and $g^{*}=0.2$.

In Fig. 9, we plot the excluded $3 \sigma$ and $5 \sigma$ discovery reaches as a function of $g^{*}$ and the top-partner mass $m_{T}$ for two typical values of integrated luminosity: 1000 and $3000 \mathrm{fb}^{-1}$. We can see that, for $m_{T}=1.0 \mathrm{TeV}$, the $5 \sigma$ level discovery sensitivities of $g^{*}$ are respectively about 0.14 with $£_{\text {int }}=1000 \mathrm{fb}^{-1}$ and 0.2 with $£_{\text {int }}=3000 \mathrm{fb}^{-1}$. On the other hand, the upper limits on the size of $g^{*}$ for $m_{T}=1.0$ (1.4) $\mathrm{TeV}$ are, respectively, given as about $g^{*} \leq 0.11(0.28)$ with $£_{\text {int }}=1000 \mathrm{fb}^{-1}$ and $g^{*} \leq 0.14(0.38)$ with $£_{\text {int }}=$ $3000 \mathrm{fb}^{-1}$.

Note that our results are obtained from simulations of a simplified model implementation which in particular fixes the branching fractions between the different top-partner decay channels, while the branching ratios can be altered in other general NP models including the $T$-quark. In Fig. 10, we plot their projected sensitivity in terms of the production cross section times branching ratio $\left(\sigma_{T} * \mathrm{Br}\right)$ for two decay channels. For the $T \rightarrow W b$ channel, we find that the single production cross sections of $\sigma_{T} * B r(T \rightarrow W b) \sim 40-50$ $\mathrm{fb}$ could be discovered at the $14 \mathrm{TeV}$ LHC with $100 \mathrm{fb}^{-1}$ for $m_{T} \in[900,1800] \mathrm{GeV}$, while the cross sections $\sim 15-20 \mathrm{fb}$ will be excluded at the $14 \mathrm{TeV}$ LHC with $100 \mathrm{fb}^{-1}$. For the $T \rightarrow t Z$ channel, we find that the single production cross sections of $\sigma_{T} * \operatorname{Br}(T \rightarrow t Z) \sim 15-45 \mathrm{fb}$ could be discovered at the $14 \mathrm{TeV}$ LHC with $3000 \mathrm{fb}^{-1}$ for $m_{T} \in[900,1400]$ $\mathrm{GeV}$, while the cross sections $\sim 5-15 \mathrm{fb}$ will be excluded.

We can now draw a comparison with other complementary studies for searches at the LHC run II involving a singlet top partner. In Ref. [80], the authors show that a mass reconstruction is possible within the $T \rightarrow t h$ decay channel at $\sqrt{s}=14 \mathrm{TeV}$ with $100 \mathrm{fb}^{-1}$ of integrated luminosity, proposing a search strategy optimized for two typical top-partner mass points, namely $m_{T}=800,900 \mathrm{GeV}$, and assuming $\operatorname{Br}(T \rightarrow t h)=100 \%$. Furthermore, the authors in Ref. [81] designed a dedicated search strategy for the leptonic 

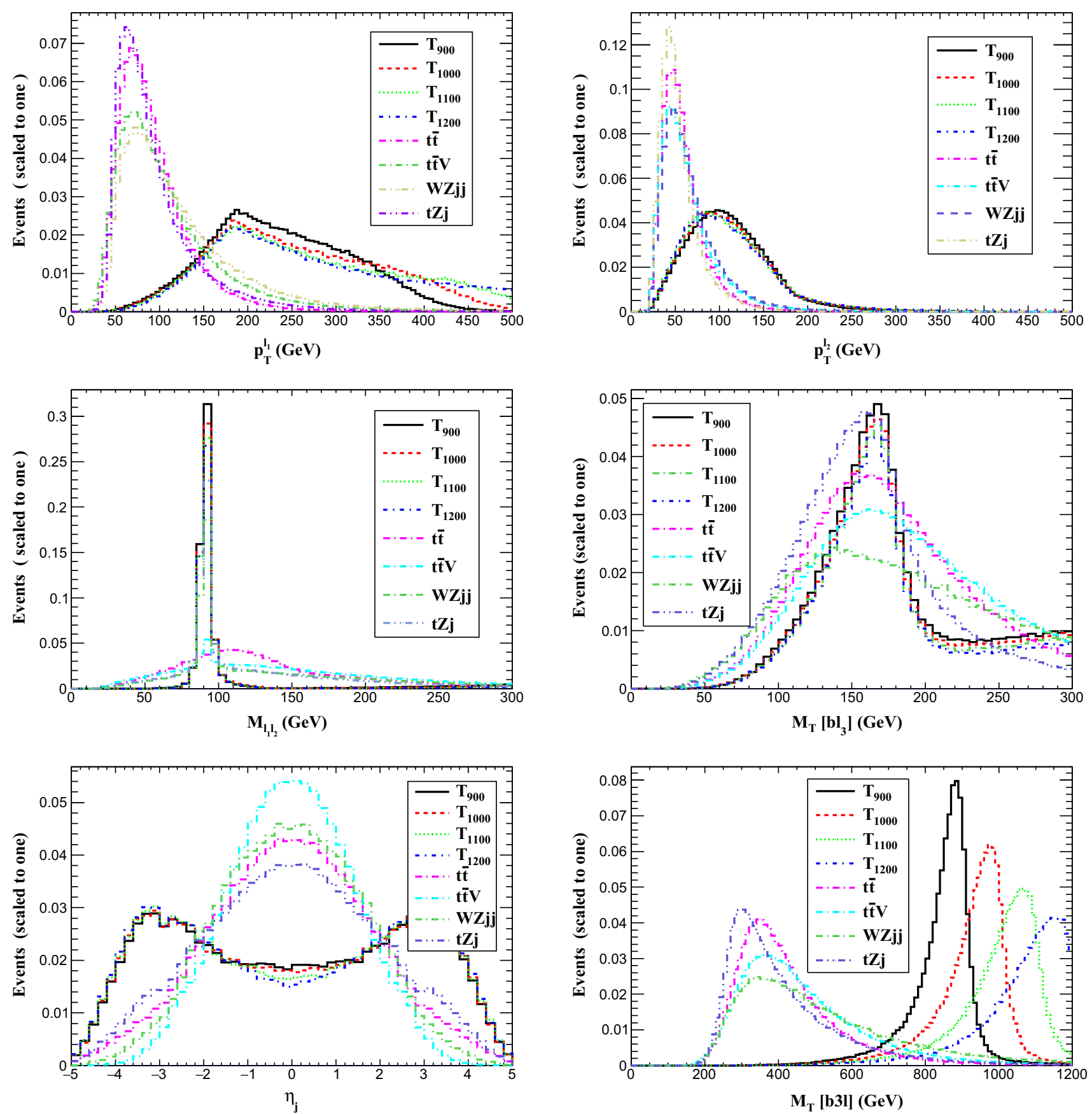

Fig. 8 Normalized distributions for the signals and backgrounds

$T \rightarrow b W$ decay channel at $\sqrt{s}=14 \mathrm{TeV}$ with $30 \mathrm{fb}^{-1}$ of integrated luminosity, obtaining an expected exclusion reach for masses up to $1.0 \mathrm{TeV}$, including both pair and single production. For two typical top-partner masses, $m_{T}=1.0$ (1.5) $\mathrm{TeV}$, the authors in Ref. [114] studied the search strategies of the single top-partner production with all the possible decay modes (i.e., $t Z, t h$ and $W b$ ) at the LHC for $\sqrt{s}=14 \mathrm{TeV}$. The results show that, for the specific model implementa- tion discussed in Ref. [114], the production cross sections of $\sigma_{T} \sim 70-140$ (30-65) fb for $m_{T}=1$ (1.5) TeV, respectively, could be discovered at the LHC with $100 \mathrm{fb}^{-1}$. Similarly, the cross sections of $\sigma_{T} \sim 27-60$ (13-24) fb for $m_{T}=1$ (1.5) $\mathrm{TeV}$, respectively, can be excluded. Therefore, our analysis is competitive with the results of the existing literature and represents a complementary candidate to search for a possible singlet top partner. 
Table 2 The cut flow of the cross sections (in fb) for four typical signals and the relevant backgrounds at the $14 \mathrm{TeV}$ LHC. Here we take the parameter as $g^{*}=0.2$

\begin{tabular}{|c|c|c|c|c|c|c|c|c|}
\hline \multirow[t]{2}{*}{ Cuts } & \multicolumn{4}{|l|}{ Signal } & \multirow[t]{2}{*}{$t \bar{t}$} & \multirow[t]{2}{*}{$t \bar{t} V$} & \multirow[t]{2}{*}{$W Z j j$} & \multirow[t]{2}{*}{$t Z j$} \\
\hline & $900 \mathrm{GeV}$ & $1000 \mathrm{GeV}$ & $1100 \mathrm{GeV}$ & $1200 \mathrm{GeV}$ & & & & \\
\hline Basic cuts & 0.49 & 0.33 & 0.21 & 0.087 & 14814 & 1.52 & 36.44 & 2.66 \\
\hline Cut-1 & 0.068 & 0.036 & 0.02 & 0.008 & 0.58 & 0.16 & 0.84 & 0.53 \\
\hline Cut-2 & 0.046 & 0.024 & 0.013 & 0.0052 & 0.0096 & 0.011 & 0.12 & 0.029 \\
\hline Cut-3 & 0.041 & 0.021 & 0.012 & 0.0047 & 0.0048 & 0.005 & 0.039 & 0.023 \\
\hline Cut-4 & 0.023 & 0.012 & 0.0065 & 0.0027 & 0.001 & $6.5 \times 10^{-4}$ & 0.0072 & 0.0052 \\
\hline Cut-5 & 0.02 & 0.011 & 0.006 & 0.0026 & $2.1 \times 10^{-4}$ & $9.2 \times 10^{-5}$ & $7.2 \times 10^{-4}$ & $9.3 \times 10^{-4}$ \\
\hline
\end{tabular}
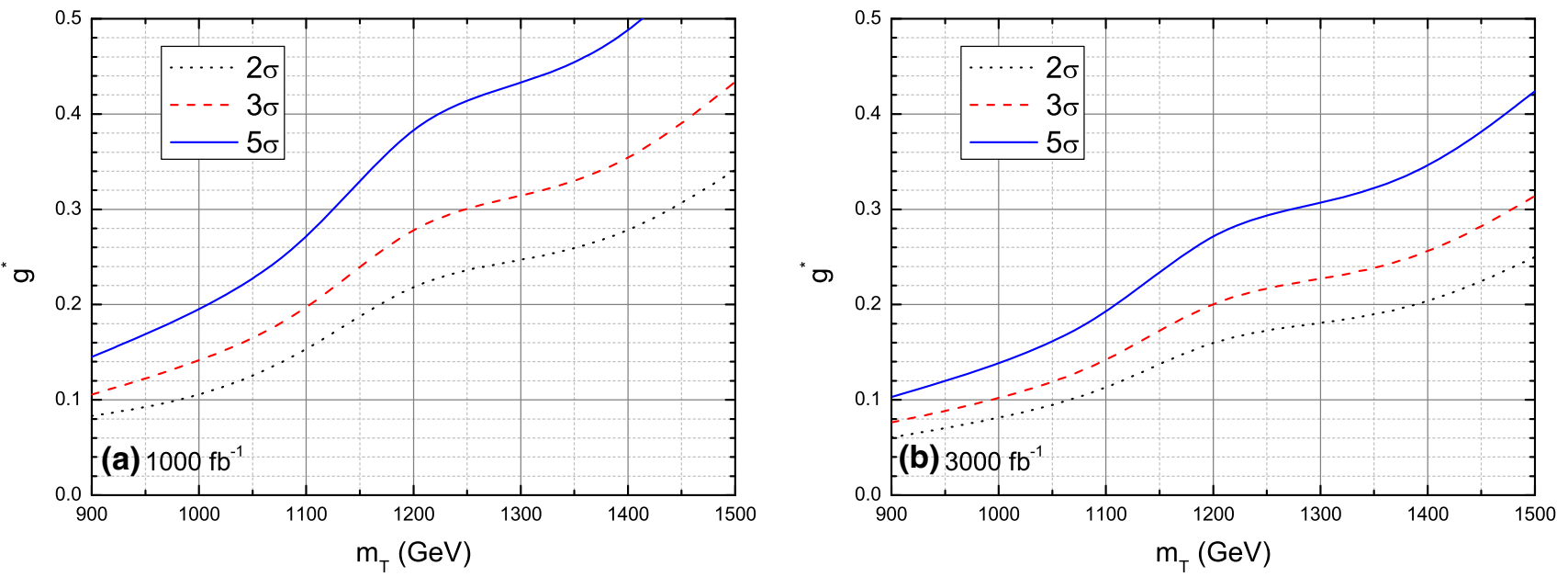

Fig. 9 The $2 \sigma, 3 \sigma$ and $5 \sigma$ contour plots for the signal in $g^{*}-m_{T}$ at $14 \mathrm{TeV}$ LHC with two typical values of integrated luminosity: a $1000 \mathrm{fb}^{-1}$, b $3000 \mathrm{fb}^{-1}$
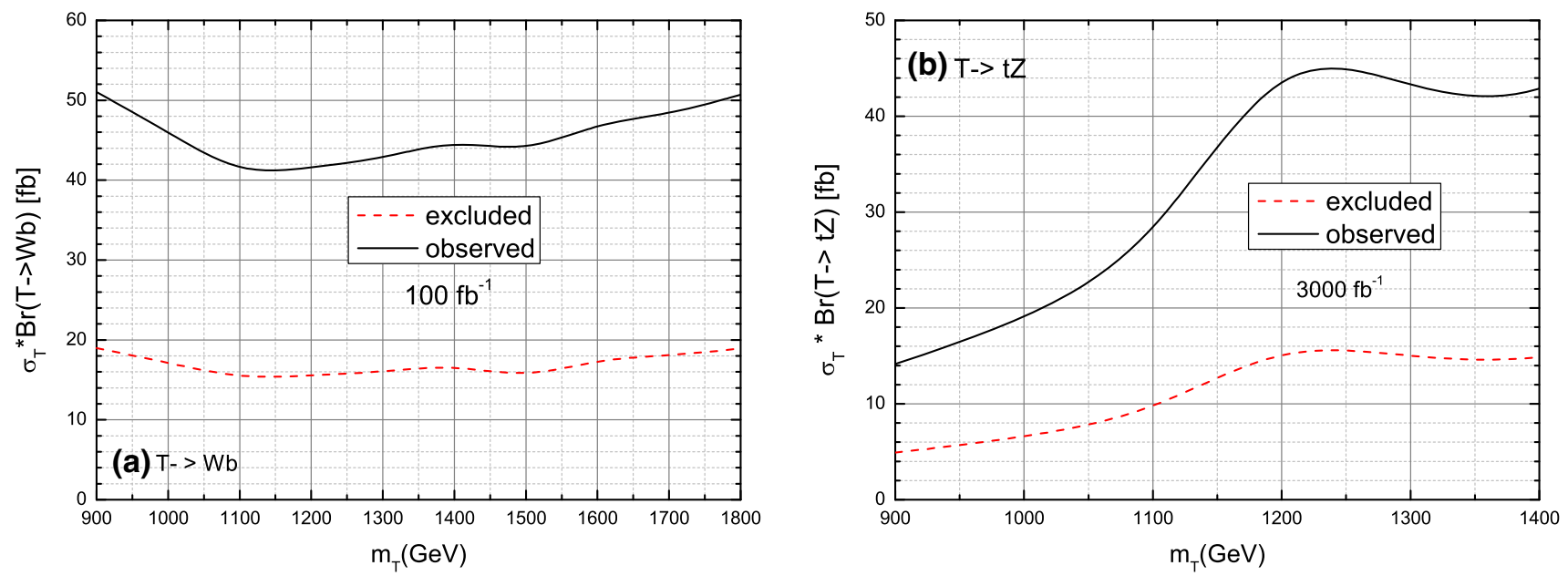

Fig. 10 The excluded and observed cross section for the signal as a function of the vector-like top partner $m_{T}$ at $14 \mathrm{TeV}$ LHC for a $T \rightarrow W b$ and b $T \rightarrow t Z$ channels

\section{Conclusion}

In this paper, we have studied the prospects of observing the single $T$ production at the $14 \mathrm{TeV}$ LHC in the $b W$ and $t Z$ decay channels. To illustrate our results, we adopt a sim- plified model including a $S U(2)_{L}$ singlet with charge $2 / 3$ with only two free parameters, namely the $T W b$ coupling parameter $g^{*}$ and the top-partner mass $m_{T}$. Since the single top-partner production depends on the $T W b$ coupling parameter $g^{*}$ and the top-partner mass $m_{T}$, the $2 \sigma$ exclusion 
limits, $3 \sigma$ evidence and the $5 \sigma$ discovery reach in the parameter plane of $g^{*}-m_{T}$ are obtained for various of integrated luminosity at the LHC Run II. In the $T \rightarrow b W \rightarrow b \ell v$ decay channel, we rely on the large transverse momentum of the $b$-jet, the lepton, and the forward nature of the light jet to suppress the backgrounds. In the $T \rightarrow t Z$ decay channel, although the leptonic decay of $Z$ entails a large suppression from the $Z$ leptonic branching ratio, the clean multilepton final state allows one to strongly reduce the backgrounds and to reconstruct the top-partner mass with high luminosity.

Even though we work in a simplified model including the singlet vector-like top partner, our results can also be mapped within the context of the specific models where the heavy $T$-quark only has couplings to the third generation of SM quarks, such as the minimal composite Higgs model [74] and the littlest Higgs model with T-parity [149]. We present a detailed analysis of their projected sensitivity in terms of the production cross section times branching fraction for the relevant decay. At the $14 \mathrm{TeV}$ LHC with $100 \mathrm{fb}^{-1}$, we find that the single production cross sections of $\sigma_{T} * \operatorname{Br}(T \rightarrow W b) \sim$ $40-50 \mathrm{fb}$ could be discovered for $m_{T} \in[900,1800] \mathrm{GeV}$, while the cross sections $\sim 15-20 \mathrm{fb}$ will be excluded. For the $T \rightarrow t Z$ channel, we find that the single production cross sections of $\sigma_{T} * \operatorname{Br}(T \rightarrow t Z) \sim 15-45 \mathrm{fb}$ could be discovered at the $14 \mathrm{TeV}$ LHC with $3000 \mathrm{fb}^{-1}$ for $m_{T} \in[900,1400]$ $\mathrm{GeV}$, while the cross sections $\sim 5-15 \mathrm{fb}$ will be excluded. We expect our analysis to represent a complementary candidate to pursue the search and mass measurement of a possible singlet top partner at the $14 \mathrm{TeV}$ LHC.

Acknowledgements This work is supported by the Joint Funds of the National Natural Science Foundation of China (Grant no. U1304112), the Foundation of Henan Educational Committee (Grant no. 2015GGJS-059) and the Foundation of Henan Institute of Science and Technology (Grant no. 2016ZD01).

Open Access This article is distributed under the terms of the Creative Commons Attribution 4.0 International License (http://creativecomm ons.org/licenses/by/4.0/), which permits unrestricted use, distribution, and reproduction in any medium, provided you give appropriate credit to the original author(s) and the source, provide a link to the Creative Commons license, and indicate if changes were made. Funded by SCOAP ${ }^{3}$.

\section{References}

1. For a review, see, P. H. Frampton, P. Q. Hung, M. Sher, Phys. Rep. 330, 263 (2000)

2. N. Arkani-Hamed, A.G. Cohen, E. Katz, A.E. Nelson, JHEP 07, 034 (2002)

3. N. Arkani-Hamed, A.G. Cohen, E. Katz, A.E. Nelson, T. Gregoire, J.G. Wacker, JHEP 08, 021 (2002)

4. K. Agashe, G. Perez, A. Soni, Phys. Rev. D 75, 015002 (2007)

5. K. Agashe, R. Contino, A. Pomarol, Nucl. Phys. B 719, 165 (2005)

6. M. Low, A. Tesi, L.-T. Wang, Phys. Rev. D 91, 095012 (2015)

7. G. Aad et al., ATLAS Collaboration. Phys. Lett. B 716, 1 (2012)
8. S. Chatrchyan et al., CMS Collaboration. Phys. Lett. B 716, 30 (2012)

9. O. Eberhardt, G. Herbert, H. Lacker, A. Lenz, A. Menzel, U. Nierste, M. Wiebusch, Phys. Rev. Lett. 109, 241802 (2012)

10. J.A. Aguilar-Saavedra, R. Benbrik, S. Heinemeyer, M. PerezVictoria, Phys. Rev. D 88, 094010 (2013)

11. A. Atre, M. Chala, J. Santiago, JHEP 1305, 099 (2013)

12. C.-Y. Chen, S. Dawson, I.M. Lewis, Phys. Rev. D 90, 035016 (2014)

13. X.-F. Wang, C. Du, H.-J. He, Phys. Lett. B 723, 314 (2013)

14. T. Abe, M. Chen, H.-J. He, JHEP 1301, 082 (2013)

15. S. Fichet, G. Moreau, Nucl. Phys. B 905, 391 (2016)

16. A. Angelescu, A. Djouadi, G. Moreau, Eur. Phys. J. C 76, 99 (2016)

17. M.-L. Xiao, J.-H. Yu, Phys. Rev. D 90, 014007 (2014)

18. S. Dawson, E. Furlan, Phys. Rev. D 89, 015012 (2014)

19. L. Lavoura, J.P. Silva, Phys. Rev. D 47, 2046 (1993)

20. C. Balazs, H.-J. He, C.-P. Yuan, Phys. Rev. D 60, 114001 (1999)

21. H.-J. He, N. Polonsky, S. Su, Phys. Rev. D 64, 053004 (2001)

22. J.A. Aguilar-Saavedra, Phys. Lett. B 625, 234 (2005)

23. J.A. Aguilar-Saavedra, G. Cynolter, E. Lendvai, Eur. Phys. J. C 58, 463 (2008)

24. J.A. Aguilar-Saavedra, G. Cynolter, E. Lendvai, JHEP 0911, 030 (2009)

25. P. Meade, M. Reece, Phys. Rev. D 74, 015010 (2006)

26. R. Contino, G. Servant, JHEP 0806, 026 (2008)

27. M.M. Nojiri, M. Takeuchi, JHEP 0810, 025 (2008)

28. J. Alwall, J.L. Feng, J. Kumar, S. Su, Phys. Rev. D 81, 114027 (2010)

29. G. Cacciapaglia, A. Deandrea, D. Harada, Y. Okada, JHEP 1011, 159 (2010)

30. J. Berger, J. Hubisz, M. Perelstein, JHEP 1207, 016 (2012)

31. Y. Okada, L. Panizzi, Adv. High Energy Phys. 2013, 364936 (2013)

32. H.-J. He, Z.-Z. Xianyu, JCAP 1410, 019 (2014)

33. S.-F. Ge, H.-J. He, J. Ren, Z.-Z. Xianyu, Phys. Lett. B 757, 480 (2016)

34. H.-C. Cheng, I. Low, L.-T. Wang, Phys. Rev. D 74, 055001 (2006)

35. S. Matsumoto, M.M. Nojiri, D. Nomura, Phys. Rev. D 75, 055006 (2007)

36. Q.-H. Cao, C.S. Li, C.-P. Yuan, Phys. Lett. B 668, 24 (2008)

37. C.-X. Yue, H.-D. Yang, W. Ma, Nucl. Phys. B 818, 1 (2009)

38. C.-Y. Chen, A. Freitas, T. Han, K.S.M. Lee, JHEP 1211, 124 (2012)

39. J. Kearney, A. Pierce, J. Thaler, JHEP 1310, 230 (2013)

40. G. Burdman, Z. Chacko, R. Harnik, L. de Lima, C.B. Verhaaren, Phys. Rev. D 91, 055007 (2015)

41. N. Liu, L. Wu, B.-F. Yang, M.-C. Zhang, Phys. Lett. B 753, 664 (2016)

42. G. Dissertori, E. Furlan, F. Moortgat, P. Nef, JHEP 1009, 019 (2010)

43. N. Chen, H.-J. He, JHEP 1204, 062 (2012)

44. O. Matsedonskyi, G. Panico, A. Wulzer, JHEP 1301, 164 (2013)

45. T. Flacke, J.H. Kim, S.J. Lee, S.H. Lim, JHEP 1405, 123 (2014)

46. J. Serra, JHEP 1509, 176 (2015)

47. T. DeGrand, Y. Shamir, Phys. Rev. D 92, 075039 (2015)

48. O. Matsedonskyi, G. Panico, A. Wulzer, JHEP 1604, 003 (2016)

49. H.-S. Goh, C.A. Krenke, Phys. Rev. D 81, 055008 (2010)

50. Y.-B. Liu, Z.-J. Xiao, Nucl. Phys. B 892, 63 (2015)

51. H.-C. Cheng, S. Jung, E. Salvioni, Y. Tsai, JHEP 1603, 074 (2016)

52. C.-H. Chen, T. Nomura, Phys. Rev. D 94, 035001 (2016)

53. S. Moretti, D. O’Brien, L. Panizzi, H. Prager, arXiv:1603.09237 [hep-ph]

54. M. Endo, Y. Takaesu, Phys. Lett. B 758, 355 (2016)

55. E.L. Berger, Q.-H. Cao, Phys. Rev. D 81, 035006 (2010)

56. B. Holdom, Q.-S. Yan, Phys. Rev. D 83, 114031 (2011) 
57. B. Holdom, Q.-S. Yan, Phys. Rev. D 84, 094012 (2011)

58. S. Yang, J. Jiang, Q.-S. Yan, X. Zhao, JHEP 1409, 035 (2014)

59. S. Gopalakrishna, T. Mandal, S. Mitra, G. Moreau, JHEP 1408, 079 (2014)

60. C. Han, A. Kobakhidze, N. Liu, L. Wu, B. Yang, Nucl. Phys. B 890, 388 (2014)

61. S.A.R. Ellis, R.M. Godbole, S. Gopalakrishna, J.D. Wells, JHEP 1409, $130(2014)$

62. Q.-H. Cao, D.-M. Zhang, arXiv:1611.09337 [hep-ph]

63. N. Arkani-Hamed, T. Han, M. Mangano, L.-T. Wang, Phys. Rep. 652, 1 (2016)

64. S. Banerjee, D. Barducci, G. Bélanger, C. Delaunay, JHEP 1611, 154 (2016)

65. M. Backović, T. Flacke, S.J. Lee, G. Perez, JHEP 1509, 022 (2015)

66. N. Vignaroli, JHEP 1207, 158 (2012)

67. N. Vignaroli, Phys. Rev. D 86, 075017 (2012)

68. G. Cacciapaglia, H. Cai, A. Carvalho, A. Deandrea, T. Flacke, B. Fuks, D. Majumder, H.-S. Shao, JHEP 1707, 005 (2017)

69. W.-Y. Keung, I. Low, Y. Zhang, Phys. Rev. D 96, 015008 (2017)

70. M. Hashimoto, Phys. Rev. D 96, 035020 (2017)

71. Y. Kats, M. McCullough, G. Perez, Y. Soreq, J. Thaler, JHEP 1706, 126 (2017)

72. G. Couture, M. Frank, C. Hamzaoui, M. Toharia, Phys. Rev. D 95, 095038 (2017)

73. Yao-Bei Liu, Nucl. Phys. B 923, 312 (2017)

74. A. De Simone, O. Matsedonskyi, R. Rattazzi, A. Wulzer, JHEP 1304, 004 (2013)

75. C. Grojean, O. Matsedonskyi, G. Panico, JHEP 1310, 160 (2013)

76. D. Barducci, A. Belyaev, J. Blamey, S. Moretti, L. Panizzi, H. Prager, JHEP 1407, 142 (2014)

77. D. Barducci, A. Belyaev, M. Buchkremer, G. Cacciapaglia, A. Deandrea, S. De Curtis, J. Marrouche, S. Moretti, L. Panizzi, JHEP 1412, 080 (2014)

78. O. Matsedonskyi, G. Panico, A. Wulzer, JHEP 1412, 097 (2014)

79. S. Moretti, D. O’Brien, L. Panizzi, H. Prager, Phys. Rev. D 96, 035033 (2017)

80. M. Endo, K. Hamaguchi, K. Ishikawa, M. Stoll, Phys. Rev. D 90, 055027 (2014)

81. B. Gripaios, T. Mueller, M.A. Parker, D. Sutherland, JHEP 1408 , 171 (2014)

82. ATLAS Collaboration, JHEP 1510, 150 (2015)

83. ATLAS Collaboration, JHEP 1508, 105 (2015)

84. CMS collaboration, Phys. Lett. B 729, 149 (2014)

85. C.M.S. Collaboration, Phys. Rev. D 93, 012003 (2016)

86. CMS Collaboration, CMS-PAS-B2G-16-002

87. ATLAS Collaboration, ATLAS-CONF-2016-013

88. ATLAS Collaboration, ATLAS-CONF-2016-101

89. ATLAS Collaboration, ATLAS-CONF-2017-015

90. H.-J. He, T.M.P. Tait, C.-P. Yuan, Phys. Rev. D 62, 011702 (2000)

91. H.-J. He, C.T. Hill, T.M.P. Tait, Phys. Rev. D 65, 055006 (2002)

92. J. Hubisz, P. Meade, A. Noble, M. Perelstein, JHEP 0601, 135 (2006)

93. F. Boudjema, A. Djouadi, C. Verzegnassi, Phys. Lett. B 238, 423 (1990)

94. R.S. Chivukula, B. Coleppa, S.D. Chiara, E.H. Simmons, H.-J. He, M. Kurachi, M. Tanabashi, Phys. Rev. D 74, 075011 (2006)

95. E.L. Berger, Q.-H. Cao, I. Low, Phys. Rev. D 80, 074020 (2009)

96. J.A. Aguilar-Saavedra, R. Benbrik, S. Heinemeyer, M. PerezVictoria, Phys. Rev. D 88, 094010 (2013)

97. F. del Aguila, M. Perez-Victoria, J. Santiago, JHEP 0009, 011 (2000)

98. G. Cacciapaglia, A. Deandrea, D. Harada, Y. Okada, JHEP 1011, 159 (2010)

99. G. Cacciapaglia, A. Deandrea, L. Panizzi, N. Gaur, D. Harada, Y. Okada, JHEP 1203, 070 (2012)

100. F.J. Botella, G.C. Branco, M. Nebot, JHEP 1212, 040 (2012)
101. G. Cacciapaglia, A. Deandrea, N. Gaur, D. Harada, Y. Okada, L. Panizzi, JHEP 1509, 012 (2015)

102. K. Ishiwata, Z. Ligeti, M.B. Wise, JHEP 1510, 027 (2015)

103. A.K. Alok, S. Banerjee, D. Kumar, S.U. Sankar, D. London, Phys. Rev. D 92, 013002 (2015)

104. F.J. Botella, G.C. Branco, M. Nebot, M.N. Rebelo, J.I. SilvaMarcos, Eur. Phys. J. C 77, 408 (2017)

105. M. Buchkremer, G. Cacciapaglia, A. Deandrea, L. Panizzi, Nucl. Phys. B 876, 376 (2013)

106. C. Anastasiou, E. Furlan, J. Santiago, Phys. Rev. D 79, 075003 (2009)

107. A. Azatov, M. Salvarezza, M. Son, M. Spannowsky, Phys. Rev. D 89, 075001 (2014)

108. M. Backović, T. Flacke, S.J. Lee, G. Perez, JHEP 1509, 022 (2015)

109. M. Backović, T. Flacke, J.H. Kim, S.J. Lee, Phys. Rev. D 92, 011701 (2015)

110. N.G. Ortiz, J. Ferrando, D. Kar, M. Spannowsky, Phys. Rev. D 90, 075009 (2014)

111. S. Beauceron, G. Cacciapaglia, A. Deandrea, J.D. Ruiz-Alvarez, Phys. Rev. D 90, 115008 (2014)

112. J. Li, D. Liu, J. Shu, JHEP 1311, 047 (2013)

113. J. Reuter, M. Tonini, JHEP 1501, 088 (2015)

114. M. Backovic, T. Flacke, J.H. Kim, S.J. Lee, JHEP 1604, 014 (2016)

115. A. Atre, G. Azuelos, M. Carena, T. Han, E. Ozcan, J. Santiago, G. Unel, JHEP 1108, 080 (2011)

116. L. Basso, J. Andrea, JHEP 1502, 032 (2015)

117. Y.-B. Liu, Phys. Rev. D 95, 035013 (2017)

118. Y.-J. Zhang, L. Han, Y.-B. Liu, Phys. Lett. B 768, 241 (2017)

119. L. Han, Y.-J. Zhang, Y.-B. Liu, Phys. Lett. B 771, 106 (2017)

120. ATLAS Collaboration, ATLAS-CONF-2016-072

121. CMS Collaborations, Phys. Lett. B 722, 634 (2017)

122. G. Aad et al., ATLAS collaboration. JHEP 1411, 104 (2014)

123. G. Brooijmans, R. Contino, B. Fuks et al., arXiv:1405.1617 [hep$\mathrm{ph}]$

124. S. Chatrchyan et al., (CMS Collaboration). JHEP 1212, 035 (2012)

125. B.W. Lee, C. Quigg, H.B. Thacker, Phys. Rev. D 16, 1519 (1977)

126. A. Alloul, N.D. Christensen, C. Degrande, C. Duhr, B. Fuks, Comput. Phys. Commun. 185, 2250 (2014)

127. https://feynrules.irmp.ucl.ac.be/wiki/VLQ_tsingletvl. Accessed 10 Jan 2016

128. K.A. Olive et al., Particle data group. Chin. Phys. C 38, 090001 (2014)

129. B. Fuks, H.-S. Shao, Eur. Phys. J. C 77, 135 (2017)

130. J. Alwall, R. Frederix, S. Frixione, V. Hirschi, F. Maltoni, O. Mattelaer, H.-S. Shao, T. Stelzer, P. Torrielli, M. Zaro, JHEP 1407, 079 (2014)

131. J. Pumplin, A. Belyaev, J. Huston, D. Stump, W.K. Tung, JHEP 0602, 032 (2006)

132. T. Sjostrand, S. Mrenna, P.Z. Skands, JHEP 0605, 026 (2006)

133. J. de Favereau et al., DELPHES 3 Collaboration. JHEP 1402, 057 (2014)

134. M. Cacciari, G.P. Salam, G. Soyez, JHEP 1804, 063 (2008)

135. E. Conte, B. Fuks, G. Serret, Comput. Phys. Commun. 184, 222 (2013)

136. M. Czakon, P. Fiedler, A. Mitov, Phys. Rev. Lett. 110, 252004 (2013)

137. J. Campbell, R.K. Ellis, F. Maltoni, S. Willenbrock, Phys. Rev. D 75, 054015 (2007)

138. J.M. Campbell, R.K. Ellis, D. Rainwater, Phys. Rev. D 68, 094021 (2003)

139. J.M. Campbell, R.K. Ellis, P. Nason, G. Zabderighi, JHEP 1308, 005 (2013)

140. S. Hoeche, F. Krauss, M. Schonherr, F. Siegert, Phys. Rev. Lett. 110, 052001 (2013) 
141. S. Kallweit, J.M. Lindert, P. Maierhöfer, S. Pozzorini, M. Schönherr, JHEP 1504, 012 (2015)

142. J. Alwall, R. Frederix, S. Frixione, V. Hirschi, O. Mattelaer, H.-S. Shao, T. Stelzer, P. Torrielli, M. Zaro, JHEP 1407, 079 (2014)

143. M.L. Mangano, M. Moretti, F. Piccinini, M. Treccani, JHEP 01, 013 (2007)

144. T. Gehrmann, M. Grazzini, S. Kallweit, P. Maierhöfer, A. von Manteuffel, S. Pozzorini, D. Rathlev, L. Tancredi, Phys. Rev. Lett. 113, 212001 (2014)
145. N. Kidonakis, Phys. Rev. D 83, 091503 (2011)

146. N. Kidonakis, Phys. Rev. D 81, 054028 (2010)

147. G. Cowan, K. Cranmer, E. Gross, O. Vitells, Eur. Phys. J. C 71, 1554 (2011)

148. V. Khachatryan et al., (CMS Collaboration). Eur. Phys. J. C 74, 3060 (2014)

149. M. Blanke, A.J. Buras, A. Poschenrieder, S. Recksiegel, C. Tarantino et al., JHEP 0701, 066 (2007) 